\title{
Gut microbial taxa elevated by dietary sugar disrupt memory function
}

\author{
Emily E. Noble ${ }^{1}$, Christine A. Olson², Elizabeth Davis ${ }^{3}$, Linda Tsan³ ${ }^{3}$ Yen-Wei Chen², Ruth Schade ${ }^{1}$, Clarissa Liu ${ }^{3}$, \\ Andrea Suarez ${ }^{3}$, Roshonda B. Jones ${ }^{3}$, Claire de La Serre', Xia Yang ${ }^{2}$, Elaine Y. Hsiao $\mathbb{C}^{2}$ and Scott E. Kanoski $\mathbb{B}^{3}$
}

\begin{abstract}
Emerging evidence highlights a critical relationship between gut microbiota and neurocognitive development. Excessive consumption of sugar and other unhealthy dietary factors during early life developmental periods yields changes in the gut microbiome as well as neurocognitive impairments. However, it is unclear whether these two outcomes are functionally connected. Here we explore whether excessive early life consumption of added sugars negatively impacts memory function via the gut microbiome. Rats were given free access to a sugar-sweetened beverage (SSB) during the adolescent stage of development. Memory function and anxiety-like behavior were assessed during adulthood and gut bacterial and brain transcriptome analyses were conducted. Taxa-specific microbial enrichment experiments examined the functional relationship between sugar-induced microbiome changes and neurocognitive and brain transcriptome outcomes. Chronic early life sugar consumption impaired adult hippocampal-dependent memory function without affecting body weight or anxiety-like behavior. Adolescent SSB consumption during adolescence also altered the gut microbiome, including elevated abundance of two species in the genus Parabacteroides ( $P$. distasonis and $P$. johnsonii) that were negatively correlated with hippocampal function. Transferred enrichment of these specific bacterial taxa in adolescent rats impaired hippocampal-dependent memory during adulthood. Hippocampus transcriptome analyses revealed that early life sugar consumption altered gene expression in intracellular kinase and synaptic neurotransmitter signaling pathways, whereas Parabacteroides microbial enrichment altered gene expression in pathways associated with metabolic function, neurodegenerative disease, and dopaminergic signaling. Collectively these results identify a role for microbiota "dysbiosis" in mediating the detrimental effects of early life unhealthy dietary factors on hippocampal-dependent memory function.
\end{abstract}

\section{Introduction}

The gut microbiome has recently been implicated in modulating neurocognitive development and consequent functioning ${ }^{1-4}$. Early life developmental periods represent critical windows for the impact of indigenous gut microbes on the brain, as evidenced by the reversal of behavioral and neurochemical abnormalities in germ free rodents when inoculated with conventional microbiota

\footnotetext{
Correspondence: Elaine Y. Hsiao (ehsiao@ucla.edu) or

Scott E. Kanoski (kanoski@usc.edu)

'University of Georgia, Athens, GA, USA

${ }^{2}$ University of California, Los Angeles, CA, USA

Full list of author information is available at the end of the article
}

during early life, but not during adulthood ${ }^{5-7}$. Dietary factors are a critical determinant of gut microbiota diversity and can alter gut bacterial communities, as evident from the microbial plasticity observed in response to pre- and probiotic treatment, as well as the "dysbiosis" resulting from consuming unhealthy, yet palatable foods that are associated with obesity and metabolic disorders (e.g., Western diet; foods high in saturated fatty acids and added sugar ${ }^{8}$. In addition to altering the gut microbiota, consumption of Western dietary factors yields long-lasting memory impairments, and these effects are more pronounced when consumed during early life developmental periods vs. during

\section{(c) The Author(s) 2021}

(c) (i) Open Access This article is licensed under a Creative Commons Attribution 4.0 International License, which permits use, sharing, adaptation, distribution and reproduction cc) in any medium or format, as long as you give appropriate credit to the original author(s) and the source, provide a link to the Creative Commons license, and indicate if changes were made. The images or other third party material in this article are included in the article's Creative Commons license, unless indicated otherwise in a credit line to the material. If material is not included in the article's Creative Commons license and your intended use is not permitted by statutory regulation or exceeds the permitted use, you will need to obtain permission directly from the copyright holder. To view a copy of this license, visit http://creativecommons.org/licenses/by/4.0/. 
adulthood $^{9-11}$. Whether diet-induced changes in specific bacterial populations are functionally related to altered early life neurocognitive outcomes, however, is poorly understood.

The hippocampus, which is well known for its role in spatial and episodic memory and more recently for regulating learned and social aspects of food intake control $^{12-17}$, is particularly vulnerable to the deleterious effects of Western dietary factors ${ }^{9,18,19}$. During the juvenile and adolescent stages of development, a time when the brain is rapidly developing, consumption of diets high in saturated fat and sugar ${ }^{20-22}$ or sugar alone ${ }^{23-26}$ impairs hippocampal function while in some cases preserving memory processes that do not rely on the hippocampus. While several putative underlying mechanisms have been investigated, the precise biological pathways linking dietary factors to neurocognitive dysfunction remain largely undetermined $^{11}$. Here we aimed to determine whether sugar-induced alterations in gut microbiota during early life are causally related to hippocampal-dependent memory impairments observed during adulthood.

\section{Methods and materials \\ Experimental subjects}

Juvenile male Sprague Dawley rats (Envigo; arrival postnatal day (PN) 26-28; 50-70 g) were housed individually in standard conditions with a 12:12 light/dark cycle. All rats had ad libitum access to water and Lab Diet 5001 (PMI Nutrition International, Brentwood, MO; 29.8 \% kcal from protein, $13.4 \% \mathrm{kcal}$ from fat, $56.7 \% \mathrm{kcal}$ from carbohydrate), with modifications where noted. Treatment group sizes for Aim 1 experiments are derived from power analyses conducted in Statistica Software (V7) based on our published data, pilot data, and relevant publications in the literature. All experiments were performed in accordance with the approval of the Animal Care and Use Committee at the University of Southern California.

\section{Experiment 1}

Twenty-one juvenile male rats (PN 26-28) were divided into two groups with equal bodyweight and given ad libitum access to (1) $11 \%$ weight-by-volume (w/v) solution containing monosaccharide ratio of $65 \%$ fructose and $35 \%$ glucose in reverse osmosis-filtered water (SUG; $n=11$ ) or 2) or an extra bottle of reverse osmosis-filtered water (CTL; $n=10$ ). This solution was chosen to model commonly consumed sugar-sweetened beverages (SSBs) in humans in terms of both caloric content and monosaccharide ratio ${ }^{27}$. In addition, all rats were given ad libitum access to water and standard rat chow. Food intake, solution intake, and body weights were monitored thrice-weekly except were prohibited due to behavioral testing. At PN 60, rats underwent Novel Object in Context (NOIC) testing, to measure hippocampal-dependent episodic contextual memory. At PN 67 rats underwent anxiety-like behavior testing in the Zero Maze, followed by body composition testing at PN 70 and an intraperitoneal glucose tolerance test (IP GTT) at PN 84. All behavioral procedures were run at the same time each day (4-6 $\mathrm{h}$ into the light cycle). Investigators were blind to animal groups when scoring the behavioral tasks such that the scorers did not know which animal was in which group. Fecal and cecal samples were collected prior to sacrifice at PN 104.

In a separate cohort of juvenile male rats ( $n=6$ /group) animals were treated as above, but on PN day 60 rats were tested in the Novel Object Recognition (NOR) and Open Field (OF) tasks, with two days in between tasks. Animals were sacrificed and tissue punches were collected from the dorsal hippocampus on PN day 65. Tissue punches were flash-frozen in a beaker filled with isopentane and surrounded dry ice and then stored at $-80^{\circ} \mathrm{C}$ until further analyses.

\section{Experiment 2}

Twenty-three juvenile male rats (PN 26-28) were divided into two groups of equal bodyweight and received a gavage twice daily ( $12 \mathrm{~h}$ apart) for 7 days (only one treatment was given on day 7) of either (1) saline (SAL; $n=8)$, or (2) a cocktail of antibiotics consisting of Vancomycin $(50 \mathrm{mg} / \mathrm{kg})$, Neomycin $(100 \mathrm{mg} / \mathrm{kg})$, and Metronidazole $(100 \mathrm{mg} / \mathrm{kg})$ along with supplementation with $1 \mathrm{mg} / \mathrm{mL}$ of ampicillin in their drinking water (ABX; $n=$ 15), which is a protocol modified from ${ }^{28}$. Animals were housed in fresh, sterile cages on Day 3 of the antibiotic or saline treatment, and again switched to fresh sterile cages on Day 7 after the final gavage. All animals were maintained on sterile, autoclaved water and chow for the remainder of the experiment. Rats in the $\mathrm{ABX}$ group were given water instead of ampicillin solution on Day 7 . Animals in the $A B X$ group were further subdivided to receive either gavage of a 1:1 ratio of Parabacteroides distasonis and Parabacteroides johnsonii (PARA; $n=8$ ) or saline (SAL; $n=7)$ thirty-six hours after the last ABX treatment. To minimize potential contamination, rats were handled minimally for 14 days. Cage changes occurred once weekly at which time animals and food were weighed. Experimenters wore fresh, sterile PPE, and weigh boxes were cleaned with sterilizing solution in between each cage change. On PN 50 rats were tested in NOIC, on PN 60 rats were tested in NOR, on PN 62 rats were tested in the Zero Maze, followed by OF on PN 64 . Investigators were blind to animal groups when scoring the behavioral tasks such that the scorers did not know which animal was in which group when timing the behavior (NOIC, NOR, Zero Maze, OF). On PN 73 rats were given an IP GTT, and on PN 76 body composition was tested. Rats were sacrificed at PN 83 and dorsal 
hippocampus tissue punches and cecal samples were collected. Tissue punches were flash-frozen in a beaker filled with isopentane and surrounded by dry ice and cecal samples were placed in microcentrifuge tubes embedded in dry ice. Samples were subsequently stored at $-80^{\circ} \mathrm{C}$ until further analyses.

\section{IP glucose tolerance test (IP GTT)}

Animals were food-restricted $24 \mathrm{~h}$ prior to IP GTT. Immediately prior to the test, baseline blood glucose readings were obtained from the tail tip and recorded by a blood glucose meter (One-touch Ultra2, LifeScan Inc., Milpitas, CA). Each animal was then intraperitoneally (IP) injected with dextrose solution $(0.923 \mathrm{~g} / \mathrm{ml}$ by body weight) and tail tip blood glucose readings were obtained at $30,60,90$, and $120 \mathrm{~min}$ after IP injections, as previously described ${ }^{23}$.

\section{Zero Maze}

The Zero Maze is an elevated circular track $(63.5 \mathrm{~cm}$ fall height, $116.8 \mathrm{~cm}$ outside diameter), divided into four equal-length sections. Two sections were open with $3 \mathrm{~cm}$ high curbs, whereas the 2 other closed sections contained $17.5 \mathrm{~cm}$ high walls. Animals are placed in the maze facing the open section of the track in a room with ambient lighting for $5 \mathrm{~min}$ while the experimenter watches the animal from a monitor outside of the room. The experimenter records the total time spent in the open sections (defined as the head and front two paws in open arms), and the number of crosses into the open sections from the closed sections.

\section{The novel object in context task}

NOIC measures episodic contextual memory based on the capacity for an animal to identify which of two familiar objects it has never seen before in a specific context. Procedures were adapted from prior reports ${ }^{29,30}$. Briefly, rats are habituated to two distinct contexts on subsequent days (with the habituation order counterbalanced by the group) for 5 -min sessions: Context 1 is a semi-transparent box $(15$ in. $\mathrm{W} \times 24$ in. $\mathrm{L} \times 12$ in. $\mathrm{H}$ ) with orange stripes and Context 2 is a grey opaque box (17 in. $\mathrm{W} \times 17$ in. $\mathrm{L} \times 16$ in. $\mathrm{H}$ ) (Context identify assignments counterbalanced by the group), each context is in a separate dimly lit room, which is obtained using two desk lamps pointed toward the floor. Day 1 of NOIC begins with each animal being placed in Context 1 containing two distinct similarly sized objects placed in opposite corners: a $500 \mathrm{ml}$ jar filled with blue water (Object A) and a square glass container (Object B) (Object assignments and placement counterbalanced by the group). On day 2 of NOIC, animals are placed in Context 2 with duplicates of one of the objects. On NOIC day 3, rats are placed in Context 2 with Objects $\mathrm{A}$ and Object B. One of these objects is not novel to the rat, but its placement in Context 2 is novel. All sessions are $5 \mathrm{~min}$ long and are video recorded. Each time the rat is placed in one of the contexts, it is placed with its head facing away from both objects. The time spent investigating each object is recorded from the video recordings by an experimenter who is blinded to the treatment groups. Exploration is defined as sniffing or touching the object with the nose or forepaws. The task is scored by calculating the time spent exploring the Novel Object to the context divided by the time spent exploring both Objects A and B combined, which is the novelty or "discrimination index". Rats with an intact hippocampus will preferentially investigate the object that is novel to Context 2 , given that this object is a familiar object yet is now presented in a novel context, whereas hippocampal inactivation impairs the preferential investigation of the object novel to Context $2^{29}$.

\section{Novel object recognition}

The apparatus used for NOR is a grey opaque box (17 in. $\mathrm{W} \times 17$ in. $\mathrm{L} \times 16$ in. $\mathrm{H}$ ) placed in a dimly lit room, which is obtained using two desk lamps pointed toward the floor. Procedures are adapted from ref. ${ }^{31}$. Rats are habituated to the empty arena and conditions for $10 \mathrm{~min}$ on the day prior to testing. The novel object and the side on which the novel object is placed are counterbalanced by the group. The test begins with a 5 -min familiarization phase, where rats are placed in the center of the arena, facing away from the objects, with two identical copies of the same object to explore. The objects were either two identical cans or two identical bottles, counterbalanced by the treatment group. The objects were chosen based on preliminary studies which determined that they are equally preferred by Sprague Dawley rats. Animals are then removed from the arena and placed in the home cage for $5 \mathrm{~min}$. The arena and objects are cleaned with 10\% ethanol solution, and one of the objects in the arena is replaced with a different one (either the can or bottle, whichever the animal has not previously seen, i.e., the "novel object"). Animals are again placed in the center of the arena and allowed to explore for $3 \mathrm{~min}$. Time spent exploring the objects is recorded via video recording and analyzed using Any-maze activity tracking software (Stoelting Co., Wood Dale, IL).

\section{Open Field}

OF measures general activity level and also anxietylike behavior in the rat. A large gray bin, $60 \mathrm{~cm}(\mathrm{~L}) \times$ $56 \mathrm{~cm}(\mathrm{~W})$ is placed under diffuse even lighting (30 lux). A center zone is identified and marked in the bin $(19 \mathrm{~cm}$ $\mathrm{L} \times 17.5 \mathrm{~cm} \mathrm{~W})$. A video camera is placed directly overhead and animals are tracked using AnyMaze Software (Stoelting Co., Wood Dale, IL). Animals are placed in the center of the box facing the back wall and allowed to 
explore the arena for $10 \mathrm{~min}$ while the experimenter watches from a monitor in an adjacent room. The apparatus is cleaned with $10 \%$ ethanol after each rat is tested.

\section{Body composition}

Body composition (body fat, lean mass) was measured using LF90 time-domain nuclear magnetic resonance (Bruker NMR minispec LF 90II, Bruker Daltonics, Inc.).

\section{Bacterial transfer}

P. distasonis (ATCC 8503) was cultured under anaerobic conditions at $37{ }^{\circ} \mathrm{C}$ in Reinforced Clostridial Medium (RCM, BD Biosciences). P. johnsonii (DSM 18315) was grown in anaerobic conditions in PYG medium (modified, DSM medium 104). Cultures were authenticated by fulllength $16 \mathrm{~S}$ rRNA gene sequencing. For bacterial enrichment, $10^{9}$ colony-forming units of both $P$. distasonis and $P$. johnsonii were suspended in $500 \mu \mathrm{L}$ pre-reduced PBS and orally gavaged into antibiotic-treated rats. When coadministered, a ratio of 1:1 was used for $P$. distasonis and P. johnsonii.

\section{Gut microbiota DNA extraction and 16s rRNA gene sequencing in sugar-fed and control rats}

All samples were extracted and sequenced according to the guidelines and procedures established by the Earth Microbiome Project ${ }^{32}$. DNA was extracted from fecal and cecal samples using the MO BIO PowerSoil DNA extraction kit. Polymerase chain reaction (PCR) targeting the V4 region of the $16 \mathrm{~S}$ rRNA bacterial gene was performed with the $515 \mathrm{~F} / 806 \mathrm{R}$ primers, utilizing the protocol described in Caporaso et $\mathrm{al}^{33}$. Amplicons were barcoded and pooled in equal concentrations for sequencing. The amplicon pool was purified with the MO BIO UltraClean PCR Clean-up kit and sequenced by the $2 \times 150 \mathrm{bp}$ MiSeq platform at the Institute for Genomic Medicine at UCSD. All sequences were deposited in Qiita Study 11255 as raw FASTQ files. Sequences were demultiplexed using Qiime-1 based "split libraries" with the forward reads only dropping. Demultiplexed sequences were then trimmed evenly to $100 \mathrm{bp}$ and $150 \mathrm{bp}$ to enable comparison to other studies for meta-analyses. Trimmed sequences were matched to known OTUs at $97 \%$ identity.

\section{Gut microbiota DNA extraction and 16S rRNA gene sequencing for Parabacteroides-enriched and control rats}

Total bacterial genomic DNA was extracted from rat fecal samples $(0.25 \mathrm{~g})$ using the Qiagen DNeasy PowerSoil Kit. The library was prepared following methods from (Caporaso et $\mathrm{al}^{33}$ ). The V4 region (515F-806R) of the 16S rDNA gene was PCR amplified using individually barcoded universal primers and $30 \mathrm{ng}$ of the extracted genomic DNA. The conditions for PCR were as follows: $94^{\circ} \mathrm{C}$ for $3 \mathrm{~min}$ to denature the DNA, with 35 cycles at $94^{\circ} \mathrm{C}$ for $45 \mathrm{~s}, 50^{\circ} \mathrm{C}$ for $60 \mathrm{~s}$, and $72{ }^{\circ} \mathrm{C}$ for $90 \mathrm{~s}$, with a final extension of $10 \mathrm{~min}$ at $72^{\circ} \mathrm{C}$. The PCR reaction was set up in triplicate, and the PCR products were purified using the Qiaquick PCR purification kit (QIAGEN). The purified PCR product was pooled in equal molar concentrations quantified by nanodrop and sequenced by Laragen, Inc. using the Illumina MiSeq platform and $2 \times 250 \mathrm{bp}$ reagent kit for paired-end sequencing. Amplicon sequence variants (ASVs) were chosen after denoising with the Deblur pipeline. Taxonomy assignment and rarefaction were performed using QIIME2-2019.10.

\section{Hippocampal RNA extraction and sequencing}

Hippocampi from rats treated with or without sugar or Parabacteroides were subject to RNA-seq analysis. Total RNA was extracted according to the manufacturer's instructions using RNeasy Lipid Tissue Mini Kit (Qiagen, Hilden, Germany). Total RNA was checked for degradation in a Bioanalyzer 2100 (Agilent, Santa Clara, CA, USA). Quality was very high for all samples, and libraries were prepared from $1 \mu \mathrm{g}$ of total RNA using a NuGen Universal Plus mRNA-seq Library Prep Kit (Tecan Genomics Inc., Redwood City, CA). Final library products were quantified using the Qubit 2.0 Fluorometer (Thermo Fisher Scientific Inc., Waltham, MA, USA), and the fragment size distribution was determined with the Bioanalyzer 2100. The libraries were then pooled equimolarly, and the final pool was quantified via qPCR using the Kapa Biosystems Library Quantification Kit, according to the manufacturer's instructions. The pool was sequenced in an Illumina NextSeq 550 platform (Illumina, San Diego, CA, USA), in Single-Read 75 cycles format, obtaining about 25 million reads per sample. The preparation of the libraries and the sequencing were performed at the USC Genome Core (http://uscgenomecore. usc.edu/).

\section{RNA-seq quality control}

Data quality checks were performed using the FastQC tool (http://www.bioinformatics.babraham.ac.uk/projects/ fastqc) and low-quality reads were trimmed with Trim_Galore (http://www.bioinformatics.babraham.ac.uk/projects/ trim_galore/). RNA-seq reads passing quality control were mapped to Rattus novegicus transcriptome (Rnor6) and quantified with Salmon ${ }^{34}$. Salmon directly mapped RNAseq reads to Rat transcriptome and quantified transcript counts. Txiimport ${ }^{35}$ was used to convert transcript counts into gene counts. Potential sample outliers were detected by principal component analysis (PCA) and one control and one treatment sample from the Parabacteroides experiment were deemed outliers (Fig. S1) and removed. 
Identification of differentially expressed genes (DEGs)

DESeq $2^{36}$ were used to conduct differential gene expression analysis between sugar treatment and the corresponding controls or between Parabacteroides treatment and the corresponding controls. Lowabundance genes were filtered out and only those having a mean raw count $>1$ in more than $50 \%$ of the samples were included. Differentially expressed genes were detected by DESeq2 with default settings. Significant DEGs were defined as Benjamini-Hochberg (BH) adjusted false-discovery rate $(\mathrm{FDR})<0.05$. For heatmap visualization, genes were normalized with variance stabilization transformation implemented in DESeq2, followed by calculating a $z$-score for each gene.

\section{Pathway analyses of DEGs}

For the pathway analyses, DEGs at an unadjusted $P$ value $<0.01$ were used. Pathway enrichment analyses were conducted using enrichr ${ }^{37}$ by intersecting each signature with pathways or gene sets from $\mathrm{KEGG}^{38}$, gene ontology biological pathways, cellular component, molecular function $^{39}$, and Wikipathways ${ }^{40}$. Pathways at FDR $<0.05$ were considered significant. Unless otherwise specified, $R$ 3.5.2 was used for the analysis mentioned in the RNA sequencing section.

\section{Additional statistical methods}

Data are presented as means \pm SEM. For analytic comparisons of body weight, total food intake, and chow intake, groups were compared using repeated-measures ANOVA in Prism software (GraphPad Inc., version 8.0). Taxonomic comparisons from 16S rRNA sequencing analysis were analyzed by analysis of the composition of microbiomes (ANCOM). When significant differences were detected, Sidak post-hoc test for multiple comparisons was used. The area under the curve for the IP GTT testing was also calculated using Prism. All other statistical analyses were performed using Student's two-tailed unpaired $t$ tests in excel software (Microsoft Inc., version 15.26). Normality was confirmed prior to the utilization of parametric testing. For all analyses, statistical significance was set at $P<0.05$. A predetermined criterion for exclusion was utilized and was based on the Grubbs Outlier Test (Prism, Graphpad Inc.) using alpha $=0.05$.

\section{Results}

\section{Early life sugar consumption impairs hippocampal- dependent memory function}

Results from the NOIC task, which measures hippocampal-dependent episodic contextual memory function ${ }^{30}$, reveal that while there were no differences in total exploration time of the combined objects on days 1 or 3 of the task (Fig. 1A, B), animals fed sugar solutions in early life beginning at PN 28 had a reduced capacity to discriminate an object that was novel to a specific context when animals were tested during adulthood (PN 60), indicating impaired hippocampal function (Fig. 1C). Conversely, animals fed sugar solutions in early life performed similarly to those in the control group when tested in the novel object recognition task (NOR) (Fig. 1D), which tests object recognition memory independent of context. Notably, when performed using the current methods with a short duration between the familiarization phase and the test phase, NOR not hippocampaldependent but instead is primarily dependent on the perirhinal cortex ${ }^{30,41-43}$. These data suggest that early life dietary sugar consumption impairs performance in hippocampal-dependent contextual-based recognition memory without affecting performance in perirhinal cortex-dependent recognition memory independent of context ${ }^{23}$.

Elevated anxiety-like behavior and altered general activity levels may influence novelty exploration independent of memory effects and may therefore confound the interpretation of behavioral results. Thus, we next tested whether early life sugar consumption affects anxiety-like behavior using two different tasks designed to measure anxiety-like behavior in the rat: the elevated zero mazes and the OF task, the latter of which also assesses levels of general activity ${ }^{44}$. Early life sugar consumption had no effect on time spent in the open area or in the number of open area entries in the zero maze (Fig. 1E, F). Similarly, early life sugar had no effect on distance traveled or time spent in the center zone in the OF task (Fig. 1G, H). Together these data suggest that habitual early life sugar consumption did not increase anxiety-like behavior or general activity levels in the rats.

\section{Early life sugar consumption impairs glucose tolerance without affecting total caloric intake, body weight, or adiposity}

Given that excessive sugar consumption is associated with weight gain and metabolic deficits ${ }^{45}$, we tested whether access to a sugar solution during the adolescent phase of development would affect food intake, body weight gain, adiposity, and glucose tolerance in the rat. Early life sugar consumption had no effect on body composition during adulthood (Fig. 1I, Fig. S2A, B). Early life sugar consumption also had no effect on body weight or total kcal intake (Fig. 1J, K), which is in agreement with the previous findings ${ }^{23,26,46}$. Animals steadily increased their intake of the $11 \%$ sugar solution throughout the study (Fig. 1L) but compensated for the calories consumed in the sugar solutions by reducing their intake of dietary chow (Fig. S2C). However, animals that were fed sugar solutions during adolescence showed impaired peripheral glucose metabolism in an IP GTT (Fig. S2D). 
Novel Object in Context

A

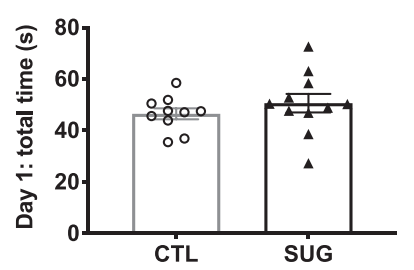

Novel Object Recognition

D

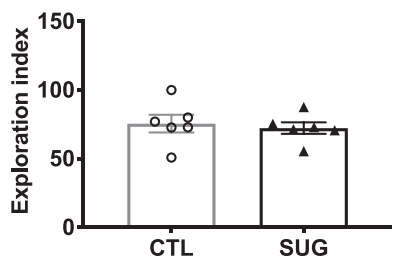

Open Field
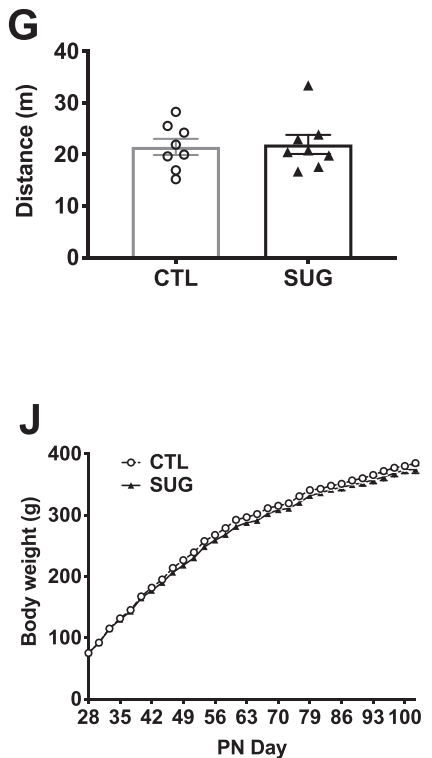

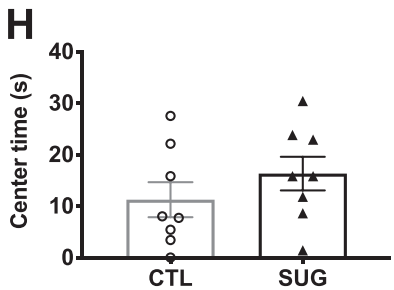

Energy Balance

B
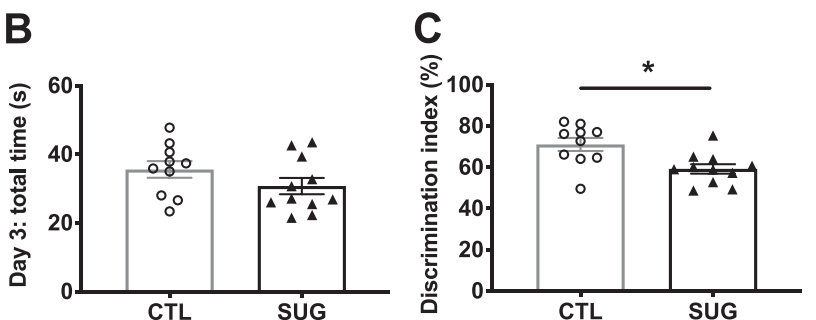

$\underline{\text { Zero Maze }}$

E
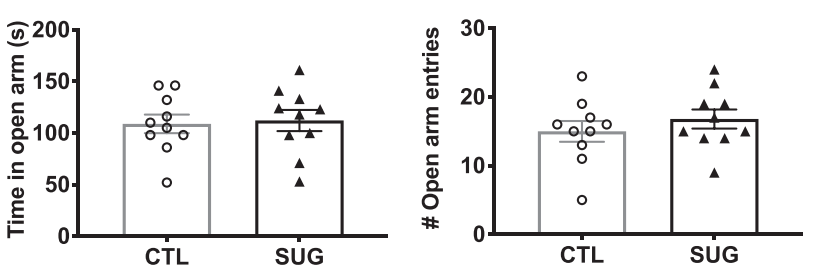

Body composition

I

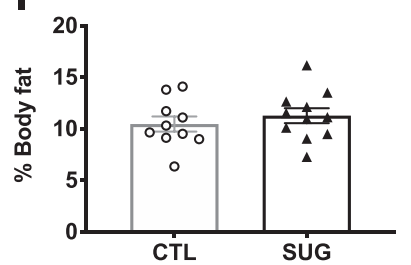

L

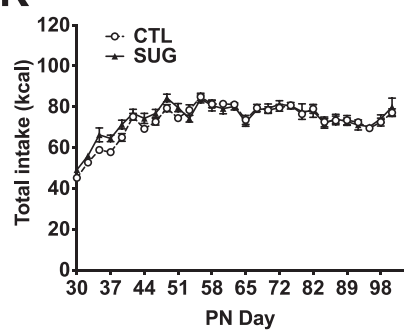

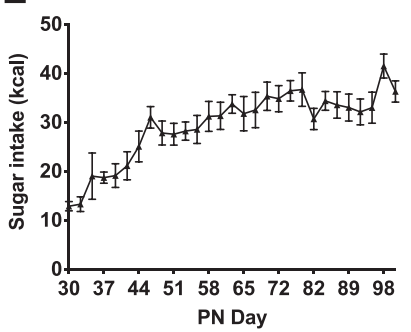

Fig. 1 Early life sugar consumption negatively impacts hippocampal-dependent memory function. A, B Early life sugar consumption had no effect on total exploration time on days 1 (familiarization) or day 3 (test day) of the Novel Object in Context (NOIC) task. C The discrimination index was significantly reduced by early life sugar consumption, indicating impaired hippocampal function $(P<0.05, n=10,11$; two-tailed, type 2 Student's $t$ test). D There were no differences in exploration index in the Novel Object Recognition (NOR task) $(n=6$; two-tailed, type 2 Student's $t$ test). E, $\mathbf{F}$ There were no differences in time spent in the open arm or the number of entries into the open arm in the Zero Maze task for anxiety-like behavior ( $n=10$; two-tailed, type 2 Student's $t$ test). $\mathbf{G}, \mathbf{H}$ There were no differences in distance traveled or time spent in the center arena in the Open Field task ( $n=8$; two-tailed, type 2 Student's $t$ test). I There was no differences in body fat \% during adulthood between rats fed early life sugar and controls ( $n=10,11$; two-tailed, type 2 Student's $t$ test). J, K Body weights and total energy intake did not differ between the groups ( $n=10,11$; two-way repeated-measures ANOVA), despite (L) increased kcal consumption from sugar-sweetened beverages in the sugar group. $C T L=$ control, SUG = sugar, PN = post-natal day; data shown as mean \pm SEM.

Gut microbiota is impacted by early life sugar consumption

Principal component analyses of $16 \mathrm{~s}$ rRNA gene sequencing data of fecal samples revealed a separation between the fecal microbiota of rats fed early life sugar and controls (Fig. 2A). Results from LEfSe analysis identified differentially abundant bacterial taxa in fecal samples that were elevated by sugar consumption. These include the family Clostridiaceae and the genus $02 d 06$ 


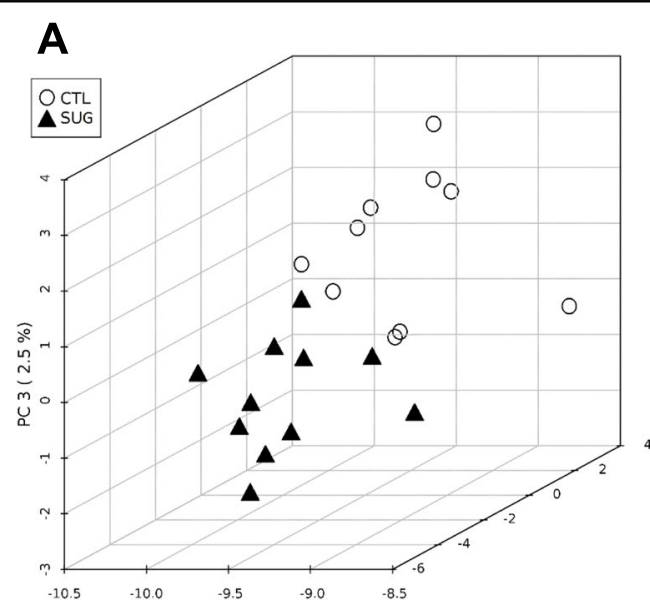

B

C

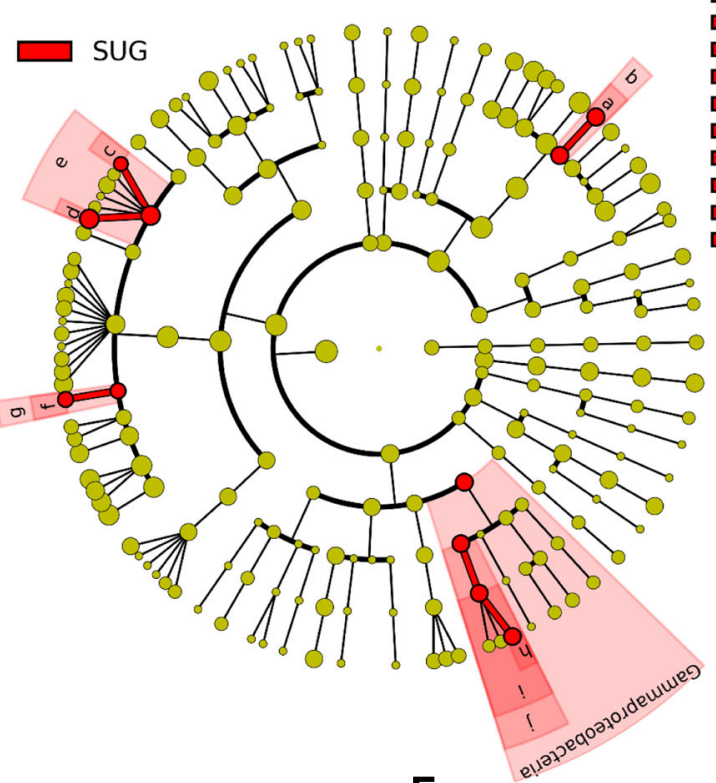

$\mathbf{E}$

$\mathbf{F}$
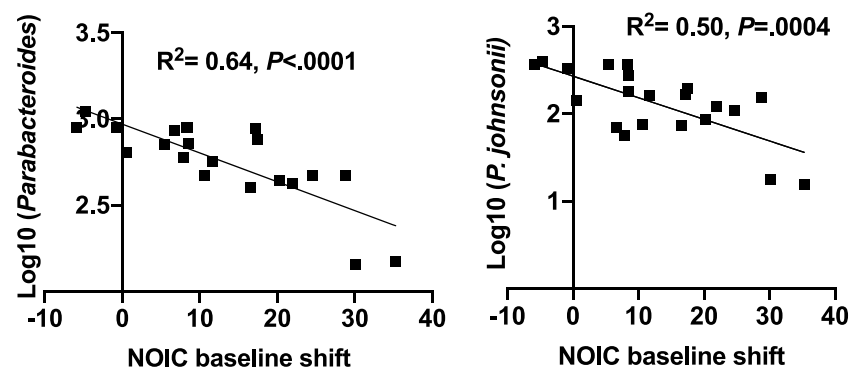

D

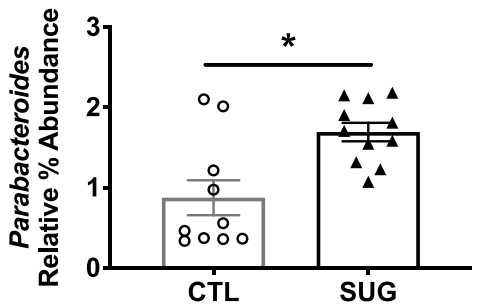

G

Fig. 2 Effect of adolescent sugar consumption on the gut microbiome in rats. A Principal component analysis showing separation between fecal microbiota of rats fed early life sugar or controls $(n=11$, 10; dark triangles = sugar, open circles $=$ control). B Results from LEfSe analysis showing Linear Discriminate Analysis (LDA) scores for microbiome analysis of fecal samples of rats fed early life sugar or controls. C A cladogram representing the results from the LEfSe analysis with the class as the outermost taxonomic level and species at the innermost level. Taxa in red are elevated in the sugar group. D Relative \% abundance of fecal Parabacteroides are significantly elevated in rats fed early life sugar $(P<0.05 ; n=11,10$, two-tailed, type 2 Student's $t$ test). $\mathbf{E}$ Linear regression of log normalized fecal Parabacteroides counts against the shift from baseline performance scores in the novel object in context task (NOIC) across all groups tested $(n=21)$. E-G Linear regression of the most abundant fecal Parabacteroides species against shift from baseline performance scores in NOIC across all groups tested $(n=21) .{ }^{*} P<0.05$; data are shown as mean \pm SEM. 
within Clostridiaceae, the family Mogibacteriaceae, the family Enterobacteriaceae, the order Enterobacteriales, the class of Gammaproteobacteria, and the genus Parabacteroides within the family Porphyromonadaceae (Fig. 2B, C). In addition to an elevated \% relative abundance of the genus Parabacteroides in animals fed early life sugar (Fig. 2D), log-transformed counts of the Parabacteroides negatively correlated with performance scores in the NOIC memory task (Fig. 2E). Of the additional bacterial populations significantly affected by sugar treatment, regression analyses did not identify any other genera as being significantly correlated to NOIC memory performance. Within Parabacteroides, levels of two operational taxonomic units (OTUs) that were elevated by sugar negatively correlated with performance in the NOIC task, identified as taxonomically related to $P$. johnsonii and $P$. distasonis (Fig. 2F, G). The significant negative correlation between NOIC performance and Parabacteroides was also present within each of the diet groups alone, but when separated out by diet group only $P$. distasonis showed a significant negative correlation for each diet group $(P<0.05)$, whereas $P$. johnsonii showed a nonsignificant trend in both the control and sugar groups $(P=0.06$, and $P=0.08$, respectively; Fig. S3A-C). The abundance of other bacterial populations that were affected by sugar consumption was not significantly related to memory task performance.

There was a similar separation between groups in bacteria analyzed from cecal samples (Fig. S4A). LEfSe results from cecal samples show elevated Bacilli, Actinobacteria, Erysipelotrichia, and Gammaproteobacteria in rats fed early life sugar, and elevated Clostridia in the controls (Fig. S4B, C). Abundances at the different taxonomic levels in fecal and cecal samples are shown in (Figs. S5 and S6). Regression analyses did not identify these altered cecal bacterial populations as being significantly correlated to NOIC memory performance.

\section{Early life Parabacteroides enrichment impairs memory function}

To determine whether neurocognitive outcomes due to early life sugar consumption could be attributable to elevated levels of Parabacteroides in the gut, we experimentally enriched the gut microbiota of naïve juvenile rats with two Parabacteroides species that exhibited high 16S rRNA sequencing alignment with OTUs that were increased by sugar consumption and were negatively correlated with behavioral outcomes in rats fed early life sugar. $P$. johnsonii and $P$. distasoni species were cultured individually under anaerobic conditions and transferred to a group of antibiotictreated young rats in a 1:1 ratio via oral gavage using the experimental design described in Methods and outlined in Fig. 3A, and from ref. ${ }^{28}$. To confirm Parabacteroides enrichment, 16SrRNA sequencing was performed on rat fecal samples for SAL-SAL, ABX-SAL, and ABX-PARA groups. Alpha diversity was analyzed using observed OTUs (Fig. 3B), where both ABX-SAL and ABX-PARA fecal samples have significantly reduced alpha diversity when compared with SAL-SAL fecal samples, suggesting that antibiotic treatment reduces microbiome alpha diversity. Further, either treatment with antibiotics alone or antibiotics followed by Parabacteroides significantly alters microbiota composition relative to the SAL-SAL group (Fig. 3C). Taxonomic comparisons from $16 \mathrm{~S}$ rRNA sequencing analysis were analyzed by analysis of the composition of microbiomes (ANCOM). Differential abundance on relative abundance at the species level (Fig. 3D) was tested across samples hypothesis-free. Significant taxa at the species level were corrected for using FDR-corrected $P$ values to calculate $W$ in ANCOM. Comparing all groups resulted in the highest $W$ value of 144 for the Parabacteroides genus, which was enriched in ABX-PARA fecal samples after bacterial gavage with an average relative abundance of $55.65 \%$ (Fig. 3E). This confirms successful Parabacteroides enrichment for ABX-PARA rats post-gavage when compared to either ABX-SAL (average relative abundance of $5.47 \%$ ) or ABX-SAL rats (average relative abundance of $0.26 \%$ ).

All rats treated with antibiotics showed a reduction in food intake and body weight during the initial stages of antibiotic treatment, however, there were no differences in body weight between the two groups of antibiotictreated animals by PN50, at the time of behavioral testing (Fig. S7A-C). Similar to a recent report ${ }^{47}$, Parabacteroides enrichment in the present study impacted body weight at later time points. Animals who received $P$. johnsonii and $P$. distasonis treatment showed reduced body weight 40 days after the transfer, with significantly lower lean mass (Fig. S7D-F). There were no differences in percent body fat between groups, nor were there significant group differences in glucose metabolism in the IPGTT (Fig. S7G). Importantly, the body weights in the ABXPARA group did not significantly differ from the ABXSAL control group at the time of behavioral testing.

Results from the hippocampal-dependent NOIC memory task showed that while there were no differences in total exploration time of the combined objects on days 1 or 3 of the task, indicating similar exploratory behavior, animals enriched with Parabacteroides showed a significantly reduced discrimination index in the NOIC task compared with either control group (Fig. 4A-C), indicating impaired performance in hippocampal-dependent memory function. When tested in the perirhinal cortexdependent NOR task $^{30}$, animals enriched with Parabacteroides showed impaired object recognition memory compared with the antibiotic-treated control group as 

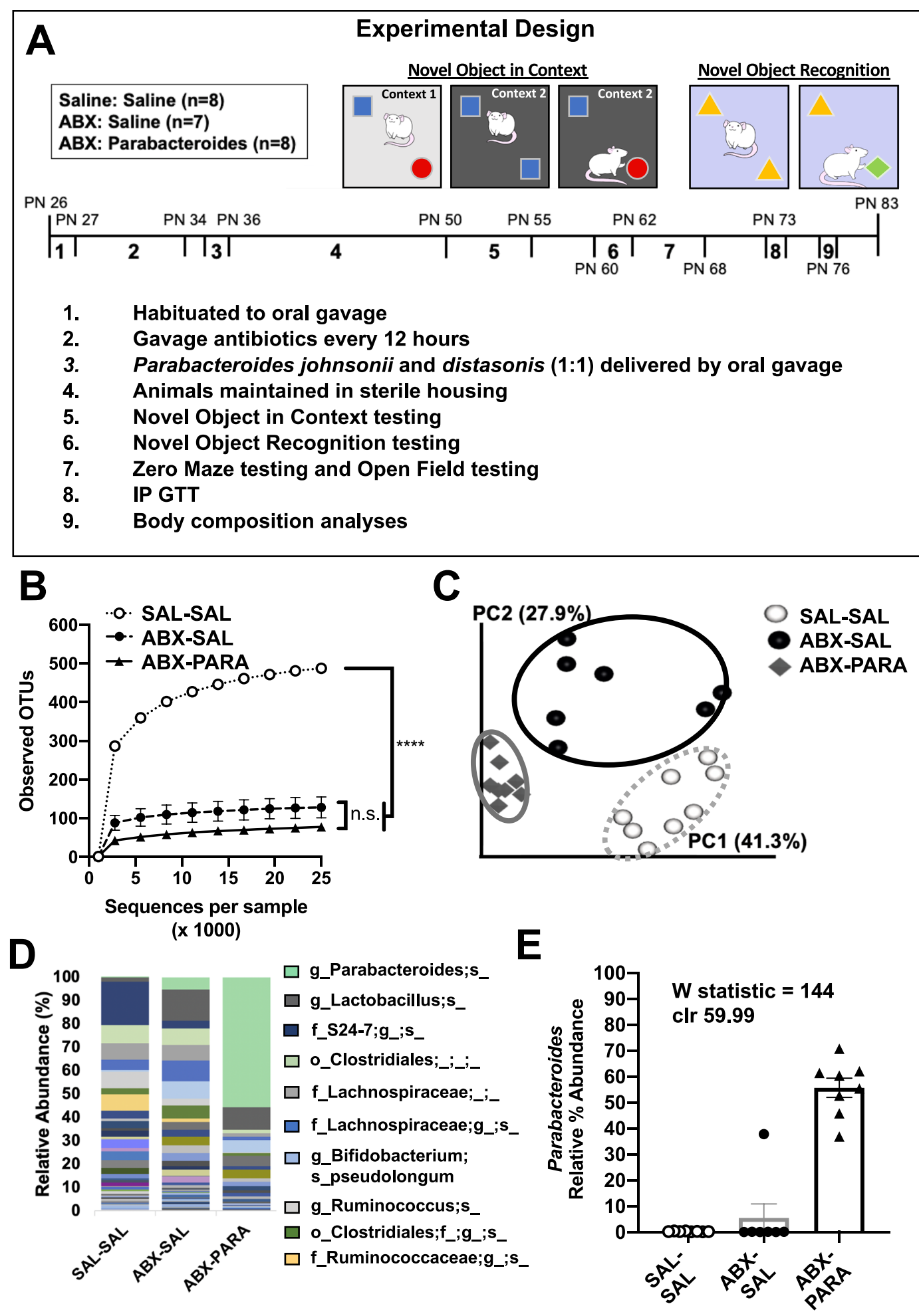

Fig. 3 (See legend on next page.) 
(see figure on previous page)

Fig. 3 Intestinal Parabacteroides is enriched by antibiotic treatment and oral gavage of $P$. distasonis and $P$. johnsonii. A Schematic showing the timeline for the experimental design of the Parabacteroides transfer experiment. B Alpha diversity based on 16S rRNA gene profiling of fecal matter $(n=7-8)$ represented by observed operational taxonomic units (OTUs) for a given number of sample sequences. $\mathbf{C}$ Principal coordinates analysis of weighted UniFrac distance based on 165 rRNA gene profiling of feces for SAL-SAL, ABX-SAL, and ABX-PARA enriched rats $(n=7-8)$. D Average taxonomic distributions of bacteria from $16 S$ rRNA gene sequencing data of feces for SAL-SAL, ABX-SAL, and ABX-PARA enriched animals $(n=7-8)$. E Relative abundances of Parabacteroides in fecal microbiota for SAL-SAL, ABX-SAL, and ABX-PARA enriched animals $(n=7-8)$ (ANCOM). PN post-natal day, IP GTT intraperitoneal glucose tolerance test. Data are presented as mean \pm S.E.M. ${ }^{*} P<0.05,{ }^{* *} P<0.01,{ }^{* * *} P<0.001$. n.s. not statistically significant, SAL-SAL rats treated with saline, ABX-SAL rats treated with antibiotics followed by sterile saline gavage, ABX-PARA rats treated with antibiotics followed by a 1:1 gavage of Parabacteroides distasonis and Parabacteroides johnsonii.

indicated by a reduced novel object exploration index (Fig. 4D). These findings show that, unlike sugar-fed animals, Parabacteroides enrichment impaired perirhinal cortex-dependent memory processes in addition to hippocampal-dependent memory.

Results from the zero maze showed no differences in time spent in the open arms nor in the number of open arm entries for the Parabacteroides-enriched rats relative to controls (Fig. 4E, F), indicating that the enrichment did not affect anxiety-like behavior. Similarly, there were no differences in distance traveled or time spent in the center arena in the OF test, which is a measure of both anxiety-like behavior and general activity in rodents (Fig. 4G, H). Together these data suggest that Parabacteroides treatment negatively impacted both hippocampal-dependent perirhinal cortex-dependent memory function without significantly affecting general activity or anxiety-like behavior.

\section{Early life sugar consumption and Parabacteroides enrichment alter hippocampal gene expression profiles}

To further investigate how sugar and Parabacteroides affect cognitive behaviors, we conducted transcriptome analysis of the hippocampus samples. Figure S1A, C shows the results of principal component analysis revealing moderate separation based on RNA sequencing data from the dorsal hippocampus of rats fed sugar in early life compared with controls. Gene pathway enrichment analyses from RNA sequencing data revealed multiple pathways significantly affected by early life sugar consumption, including four pathways involved in neurotransmitter synaptic signaling: dopaminergic, glutamatergic, cholinergic, and serotonergic signaling pathways. In addition, several gene pathways that also varied by sugar were those involved in kinase-mediated intracellular signaling: cGMP-PKG, RAS, cAMP, and MAPK signaling pathways (Fig. 5A, Table S1).

Analyses of individual genes across the entire transcriptome using a stringent FDR criterion further identified 21 genes that were differentially expressed in rats fed early life sugar compared with controls, with 11 genes elevated and 10 genes decreased in rats fed sugar compared to controls (Fig. 5B). Among the genes impacted, several genes that regulate cell survival, migration, differentiation, and DNA repair were elevated by early life sugar access, including Faap100, which encodes an FA core complex member of the DNA damage response pathway $^{48}$, and Eepd1, which transcribes an endonuclease involved in repairing stalled DNA replication forks, stressed from DNA damage ${ }^{49}$. Other genes associated with endoplasmic reticulum stress and synaptogenesis were also significantly increased by sugar consumption, including Klf9, Dgkh, Neurod2, Ppl, and Kirrel1 ${ }^{50-53}$.

Several genes were reduced by dietary sugar, including Tns2, which encodes tensin 2, important for cell migration $^{54}$, RelA, which encodes an NF/kB complex protein that regulates the activity-dependent neuronal function and synaptic plasticity ${ }^{55}$, and Grm8, the gene for the metabotropic glutamate receptor 8 (mGluR8). Notably, reduced expression of the mGluR8 receptor may contribute to the impaired neurocognitive functioning in animals fed sugar, as mGluR8 knockout mice show impaired hippocampaldependent learning and memory ${ }^{56}$.

Figure S1A, B, D shows the results of the principal component analysis of dorsal hippocampus RNA sequencing data indicating a moderate separation between rats enriched with Parabacteroides and controls. Gene pathway analyses revealed that early life Parabacteroides treatment, similar to effects associated with sugar consumption, significantly altered the genetic signature of dopaminergic synaptic signaling pathways, though differentially expressed genes were commonly affected in opposite directions between the two experimental conditions (Fig. S8). Parabacteroides treatment also impacted gene pathways associated with metabolic signaling. Specifically, pathways regulating fatty acid oxidation, rRNA metabolic processes, mitochondrial inner membrane, and valine, leucine, and isoleucine degradation were significantly affected by Parabacteroides enrichment. Other pathways that were influenced were those involved in neurodegenerative disorders, including Alzheimer's disease and Parkinson's disease, though most of the genes affected in these pathways were mitochondrial genes (Fig. 5D, Table S2).

At the level of individual genes, dorsal hippocampal RNA sequencing data revealed that 15 genes were 


\section{Novel Object in Context}

A

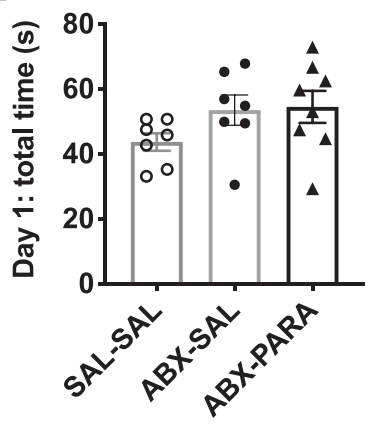

B

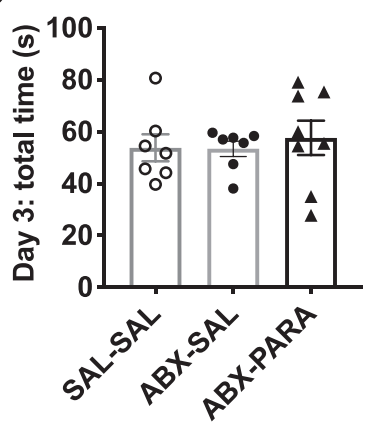

C

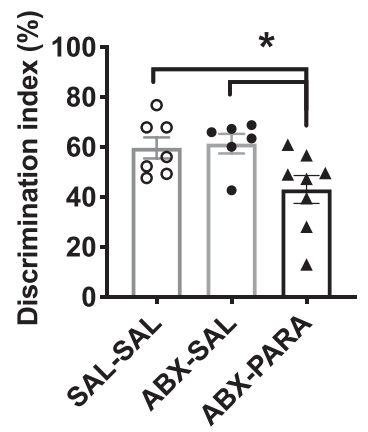

$\underline{\text { Novel Object Recognition }}$

Zero Maze

D

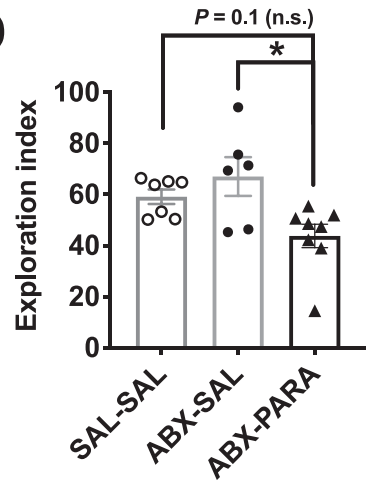

G

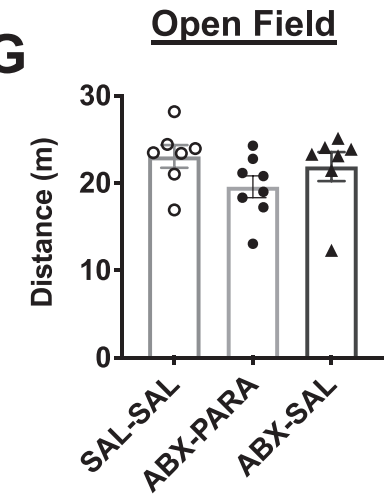

E

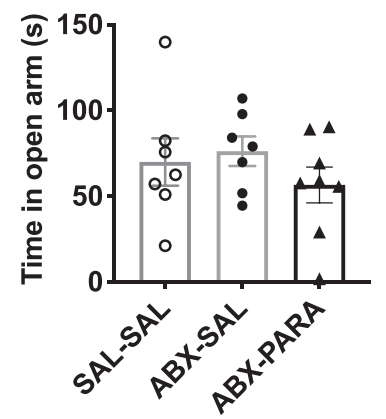

$\mathbf{F}$

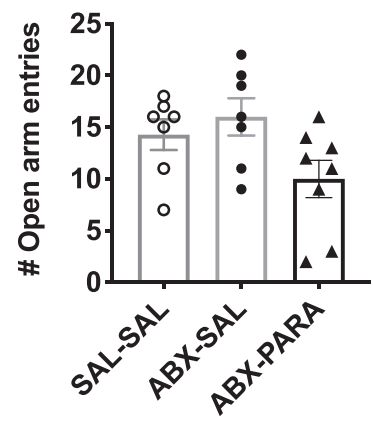

H

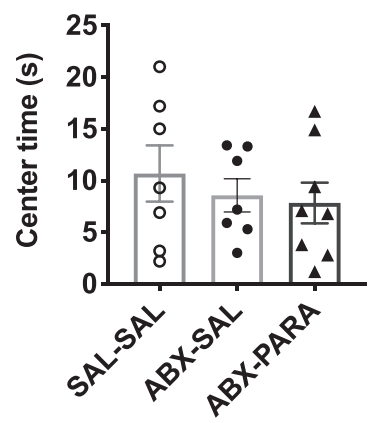

Fig. 4 Early life enrichment with Parabacteroides negatively impacts neurocognitive function. A, B Early life enrichment with a 1:1 ratio of $P$. johnsonii and $P$. distasonis had no effect on total exploration time in the Novel Object in Context (NOIC) task. C Discrimination index was significantly reduced by enrichment with $P$. johnsonii and $P$. distasonis, indicating impaired hippocampal function $\left(n=7,8 ; F_{(2,19)}=4.92 ; P<0.05\right.$, oneway ANOVA with Tukey's multiple comparison test). D There was a significant reduction in the exploration index in the Novel Object Recognition (NOR task), indicating impaired perirhinal cortex function $\left(n=7,8 ; F_{(2,19)}=3.61 ; P<0.05\right.$, one-way ANOVA with Tukey's multiple comparison test). $\mathbf{E}, \mathbf{F}$ There were no differences in time spent or a number of entries into the open arm by animals with $P$. johnsonii and $P$. distasonis enrichment in the Zero Maze task for anxiety-like behavior ( $n=7,8$; one-way ANOVA). G, $\mathbf{H}$ There were no differences in distance traveled or time spent in the center arena in the Open Field task ( $n=7,8$; one-way ANOVA). SAL-SAL saline-saline control, ABX-SAL antibiotics-saline control, ABX-PARA antibiotics-P. johnsonii and $P$. distasonis enriched, PN post-natal day; data shown as mean \pm SEM; ${ }^{*} P<0.05$. 


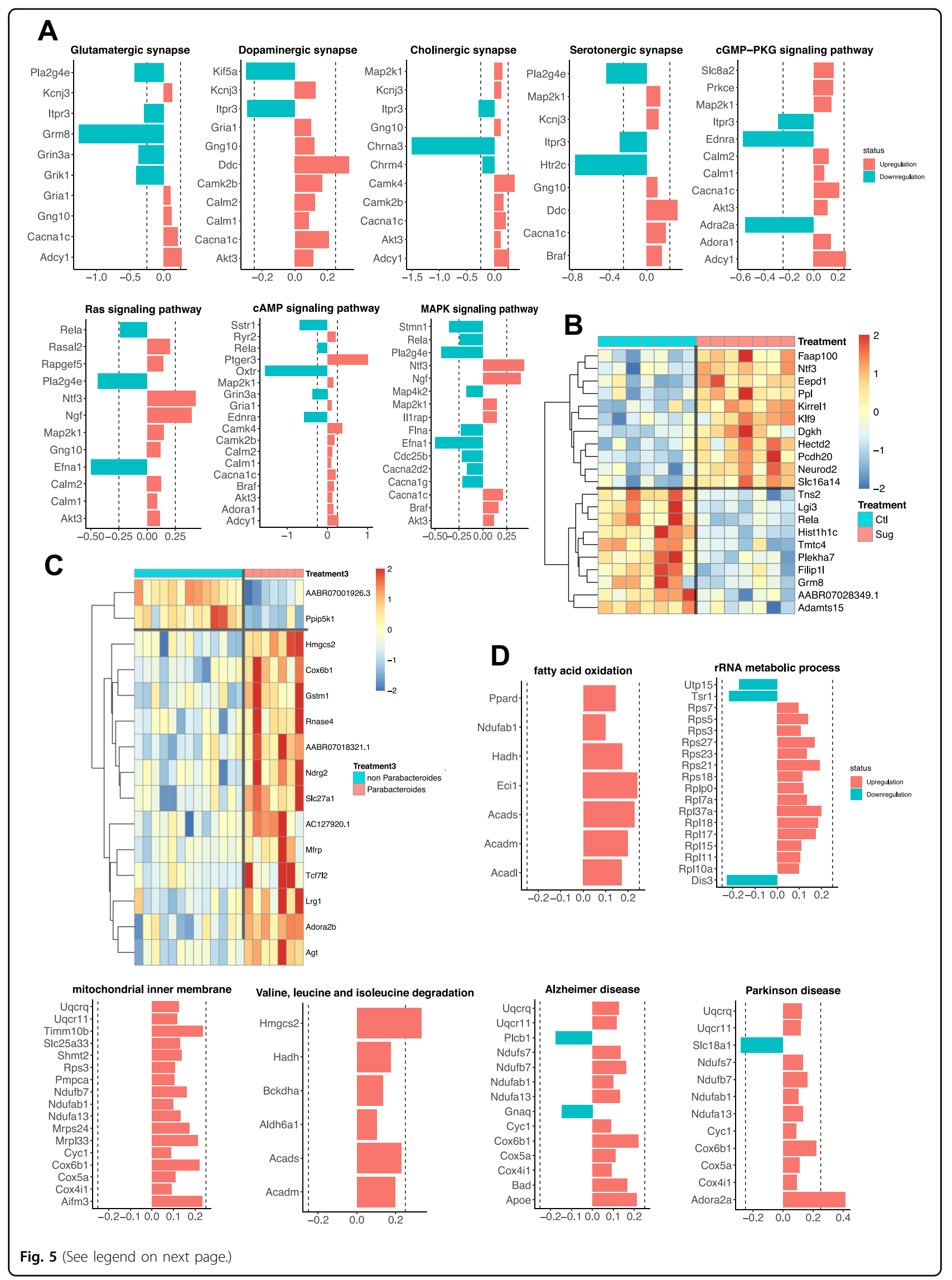


(see figure on previous page)

Fig. 5 Effect of early life sugar or targeted Parabacteroides enrichment on hippocampal gene expression. A Pathway analyses for differentially expressed genes (DEGs) at a $P$ value $<0.01$ in hippocampal tissue punches from rats fed early life sugar compared with controls. Upregulation by sugar is shown in red and downregulation by sugar in blue. B A heatmap depicting DEGs that survived the Benjamini-Hochberg corrected FDR of $P$ $<0.05$ in rats fed early life sugar compared with controls. Warmer colors (red) signify an increase in gene expression and cool colors (blue) a reduction in gene expression by treatment (CTL control, SUG early life sugar; $n=7 /$ group). C A heatmap depicting DEGs that survived the Benjamini-Hochberg corrected FDR of $P<0.05$ in rats with early life Parabacteroides enrichment compared with combined control groups. Warmer colors (red) signify an increase in gene expression and cool colors (blue) a reduction in gene expression by treatment $(n=7,14)$. D Pathway analyses for differentially expressed genes (DEGs) at a $P$ value $<0.01$ in rats enriched with Parabacteroides compared with combined controls. Upregulation by Parabacteroides transfer is shown in red and downregulation in blue. The dotted line indicates $\pm 0.25 \log 2$ fold change.

differentially expressed in rats enriched with Parabacteroides compared with controls, with 13 genes elevated and two genes decreased in the Parabacteroides group compared with controls (Fig. 5C). Consistent with results from gene pathway analyses, several individual genes involved in metabolic processes were elevated by Parabacteroides enrichment, such as Hmgcs2, which is a mitochondrial regulator of ketogenesis and provides energy to the brain under metabolically taxing conditions or when glucose availability is low ${ }^{57}$, and Cox6b1, a mitochondrial regulator of energy metabolism that improves hippocampal cellular viability following ischemia/reperfusion injury ${ }^{58}$. Parabacteroides enrichment was also associated with incased expression of Slc27A1 and $M f r p$, which are each critical for the transport of fatty acids into the brain across capillary endothelial cells ${ }^{59,60}$.

\section{Discussion}

Dietary factors are a key source of gut microbiome diversity $^{28,46,61-63}$ and emerging evidence indicates that diet-induced alterations in the gut microbiota may be linked with altered neurocognitive development ${ }^{28,63-65}$. Our results identify species within the genus Parabacteroides that are elevated by habitual early life consumption of dietary sugar and are negatively associated with hippocampal-dependent memory performance. Further, targeted microbiota enrichment of Parabacteroides perturbed both hippocampal- and perirhinal cortex-dependent memory performance. These findings are consistent with previous literature in showing that early life consumption of Western dietary factors impairs neurocognitive outcomes ${ }^{10,11}$, and further suggest that altered gut bacteria due to excessive early life sugar consumption may functionally link dietary patterns with cognitive impairment.

Our previous data show that rats are not susceptible to habitual sugar consumption-induced learning and memory impairments when $11 \%$ sugar solutions are consumed ad libitum during adulthood, in contrast to effects observed in the present and previous study in which the sugar is consumed during early life development ${ }^{23}$. It is possible that habitual sugar consumption differentially affects the gut microbiome when consumed during adolescence vs. adulthood. However, a recent report showed that adult consumption of a high fructose diet $(35 \% \mathrm{kcal}$ from fructose) promotes gut microbial "dysbiosis" and neuroinflammation and cell death in the hippocampus, yet without impacting cognitive function ${ }^{66}$, suggesting that perhaps neurocognitive function is more susceptible to gut microbiota influences during early life than during adulthood. Indeed, several reports have identified early life critical periods for microbiota influences on behavioral and neurochemical endpoints in germ-free mice ${ }^{5,75}$. However, the age-specific profile of sugar-associated microbiome dysbiosis and neurocognitive impairments remains to be determined.

Given that the adolescent rats consuming SSBs compensated for these calories by consuming less chow, it is possible that reduced nutrient (e.g., dietary protein) consumption may have contributed to the deficits in hippocampal function. However, we think this is unlikely, as adolescent SSB access did not produce any substantial nutrient deficiency that would restrict growth, as evidenced by the similarities in body weight between the experimental and control group. Furthermore, prior studies that directly examined the effects of adolescent caloric (and thereby nutrient) restriction on learning and memory in rats found that there were no differences in hippocampal-dependent memory function when rats were restricted by $\sim 40 \%$ from PN 25 to PN $67^{67}$, Importantly, the parameters in this study closely match those in the present study, as our adolescent SSB access was given over a similar developmental period prior to behavioral testing, and produced a $\sim 40 \%$ reduction in total chow kcal consumption. Thus, it is likely that excessive sugar consumption and not nutrient deficiency led to memory deficits, although future work is needed to more carefully examine these variables independently.

While our study reveals a strong negative correlation between levels of fecal Parabacteroides and performance in the hippocampal-dependent contextual episodic memory NOIC task, as well as impaired NOIC performance in rats given access to a sugar solution during adolescence, sugar intake did not produce impairments in 
the perirhinal cortex-dependent NOR memory task. This is consistent with our previous report in which rats given access to an $11 \%$ sugar solution during adolescence were impaired in hippocampal-dependent spatial memory (Barne's maze procedure), yet were not impaired in a nonspatial task of comparable difficulty that was not hippocampal-dependent ${ }^{23}$. Present results revealing that early life sugar consumption negatively impacts hippocampal-dependent contextual-based object recognition memory (NOIC) without influencing NOR memory performance is also consistent with previous reports using a cafeteria diet high in both fat content and sugar $^{68,69}$. On the other hand, enrichment of $P$. johnsonii and $P$. distasonis in the present study impaired memory performance in both tasks, suggesting a broader impact on neurocognitive functioning with this targeted bacterial enrichment approach.

Gene pathway analyses from dorsal hippocampus RNA sequencing identified multiple neurobiological pathways that may functionally connect gut dysbiosis with memory impairment. Early life sugar consumption was associated with alterations in several neurotransmitter synaptic signaling pathways (e.g., glutamatergic and cholinergic) and intracellular signaling targets (e.g., cAMP and MAPK). A different profile was observed in Parabacteroides-enriched animals, where gene pathways involved with metabolic function (e.g., fatty acid oxidation and branched-chain amino acid degradation) and neurodegenerative disease (e.g., Alzheimer's disease) were altered relative to controls. Given that sugar has effects on bacterial populations in addition to Parabacteroides, and that sugar consumption and Parabacteroides treatment differentially influenced peripheral glucose metabolism and body weight, these transcriptome differences in the hippocampus are not surprising. However, gene clusters involved with dopaminergic synaptic signaling were significantly influenced by both early life sugar consumption and Parabacteroides treatment, thus identifying a common pathway through which both diet-induced and gut bacterial infusion-based elevations in Parabacteroides may influence neurocognitive development. Though differentially expressed genes were commonly affected in opposite directions in Parabacteroides enriched animals compared with early life sugar treated animals, it is possible that perturbations to the dopamine system play a role in the observed cognitive dysfunction. For example, while dopamine signaling in the hippocampus has not traditionally been investigated for mediating memory processes, several recent reports have identified a role for dopamine inputs from the locus coeruleus in regulating hippocampal-dependent memory and neuronal activity ${ }^{70,71}$. Interestingly, endogenous dopamine signaling in the hippocampus has recently been linked with regulating food intake and food-associated contextual learning ${ }^{72}$, suggesting that dietary effects on gut microbiota may also impact feeding behavior and energy balance-relevant cognitive processes.

It is important to note that comparisons between the gene expressional analyses in the Parabacteroides enrichment and sugar consumption experiments should be made cautiously given that there were slight differences in the timing of the hippocampus tissue harvest between the two experiments (PN 65 for sugar consumption vs. PN 83 for the Parabacteroides enrichment). Further, future work is needed to determine whether differences in gene expression observed in each experiment translates to differential expression at the protein level. It is also worth emphasizing that the levels of Parabacteroides conferred by our enrichment study were substantially higher than in the dietary sugar study, and thus it is not surprising that Parabacteroides enrichment would confer a different impact on host physiology, hippocampal gene expression, and neurocognition compared to Parabacteroides elevations associated with SSB consumption. Regardless of these caveats in comparing the two models, our data extend the field by highlighting a specific bacterial population that (1) is capable of negatively impacting neurocognitive development when experimentally enriched, and (2) is elevated by early life consumption of dietary sugar with levels correlating negatively with hippocampal-dependent memory performance.

Many of the genes that were differentially upregulated in the hippocampus by Parabacteroides enrichment were involved in fat metabolism and transport. Thus, it is possible that Parabacteroides conferred an adaptation in the brain, shifting fuel preference away from carbohydrate toward lipid-derived ketones. Consistent with this framework, Parabacteroides were previously shown to be upregulated by a ketogenic diet in which carbohydrate consumption is drastically depleted and fat is used as a primary fuel source due. Furthermore, enrichment of Parabacteroides merdae together with Akkermansia muciniphila was protective against seizures in mice ${ }^{28}$. It is possible that $P$. distasonis reduces glucose uptake from the gut, enhances glucose clearing from the blood, and/or alters nutrient utilization in general, an idea further supported by the recent finding that $P$. distasonis is associated with reduced diet- and genetic-induced obesity and hyperglycemia in mice ${ }^{47}$.

The present findings produce several opportunities for further mechanistic investigation. For example, how do diet-induced alterations in gut bacteria impact the brain? Several possible mechanisms have been investigated and proposed, such as impaired gut barrier function and endotoxemia ${ }^{63,73}$, perhaps related to altered short-chain fatty acid production ${ }^{66,74}$. Moreover, it is well-known that the liver is negatively impacted by excessive fructose consumption $^{75}$, and emerging evidence highlights a gut microbiome-liver axis with crosstalk via bile acids and 
cytokines $^{76}$. It is possible that dietary sugar-induced microbiota changes alter the hepatic-gut axis, thus contributing to altered cognitive function. Indeed, an altered bile acid profile due to gut microbiota-produced bile acid secondary metabolites is associated with cognitive dysfunction in Alzheimer's Disease in humans ${ }^{77}$.

Taken together, our collective results provide insight into the neurobiological mechanisms that link early life unhealthy dietary patterns with altered gut microbiota changes and neurocognitive impairments. Currently, probiotics, live microorganisms intended to confer health benefits, are not regulated with the same rigor as pharmaceuticals but instead are sold as dietary supplements. Our findings suggest that gut enrichment with certain species of Parabacteroides is potentially harmful to neurocognitive mnemonic development. These results highlight the importance of conducting rigorous basic science analyses on the relationship between diet, microorganisms, brain, and behavior prior to widespread recommendations of bacterial microbiome interventions for humans.

\section{Acknowledgements}

We thank Alyssa Cortella for contributing the rodent artwork. We thank Caroline Szjewski, Lekha Chirala, Vaibhav Konanur, Sarah Terrill, and Ted Hsu for their critical contributions to the research. The research was supported by DK1 16942 and DK104897, and institutional funds to S.E.K., DK1 18000 and DK111158 to E.E.N., DK116558 to A.N.S., D.K. 118944 to C.M.L. C.A.O. was supported by an F31 AG064844. E.Y.H. was supported by the ARO MURI award W911NF-17-1-0402. DK104363 to X.Y., Eureka Scholarship and BWF-CHIP Fellowship to Y.C.

\section{Author details}

${ }^{1}$ University of Georgia, Athens, GA, USA. ${ }^{2}$ University of California, Los Angeles, CA, USA. ${ }^{3}$ University of Southern California, Los Angeles, CA, USA

\section{Data availability}

All data are available upon request. The $16 \mathrm{~S}$ rRNI microbiome sequencing data are available through Qiita (ID 13651 and 11255) and the RNA sequencing data are available through the NCBI Gene Expression Omnibus, GSE150091.

\section{Conflict of interest}

The authors declare no competing interests.

\section{Publisher's note}

Springer Nature remains neutral with regard to jurisdictional claims in published maps and institutional affiliations.

Supplementary information The online version contains supplementary material available at https://doi.org/10.1038/s41398-021-01309-7.

Received: 26 January 2021 Revised: 18 February 2021 Accepted: 2 March 2021

Published online: 31 March 2021

\section{References}

1. Vuong, H. E., Yano, J. M., Fung, T. C. \& Hsiao, E. Y. The microbiome and host behavior. Annu. Rev. Neurosci. 40, 21-49 (2017).

2. Noble, E. E., Hsu, T. M. \& Kanoski, S. E. Gut to brain dysbiosis: mechanisms linking western diet consumption, the microbiome, and cognitive impairment. Front. Behav. Neurosci. 11, 9 (2017).
3. Lach, G. et al. Enduring neurobehavioral effects induced by microbiota depletion during the adolescent period. Transl. Psychiatry 10, 382 (2020).

4. Morais, L. H. et al. Enduring behavioral effects induced by birth by caesarean section in the mouse. Curr. Biol. 30, 3761-3774 e3766 (2020).

5. Neufeld, K. A., Kang, N., Bienenstock, J. \& Foster, J. A. Effects of intestinal microbiota on anxiety-like behavior. Commun. Integr. Biol. 4, 492-494 (2011).

6. Sudo, N. et al. Postnatal microbial colonization programs the hypothalamicpituitary-adrenal system for stress response in mice. J. Physiol. 558, 263-275 (2004).

7. Diaz Heijtz, R. et al. Normal gut microbiota modulates brain development and behavior. Proc. Natl Acad. Sci. USA 108, 3047-3052 (2011).

8. Cryan, J. F. et al. The microbiota-gut-brain axis. Physiol. Rev. 99, 1877-2013, https://doi.org/10.1152/physrev.00018.2018 (2019).

9. Kanoski, S. E. \& Davidson, T. L. Western diet consumption and cognitive impairment: links to hippocampal dysfunction and obesity. Physiol. Behav. 103, 59-68 (2011).

10. Noble, E. E., Hsu, T. M., Liang, J. \& Kanoski, S. E. Early-life sugar consumption has long-term negative effects on memory function in male rats. Nutr. Neurosci. https://doi.org/10.1080/1028415X.2017.1378851 (2019).

11. Noble, E. E. \& Kanoski, S. E. Early life exposure to obesogenic diets and learning and memory dysfunction. Curr. Opin. Behav. Sci. 9, 7-14 (2016).

12. Hsu, T. M. et al. Hippocampus ghrelin receptor signaling promotes sociallymediated learned food preference. Neuropharmacology 131, 487-496 (2018).

13. Hsu, T. M. et al. A hippocampus to prefrontal cortex neural pathway inhibits food motivation through glucagon-like peptide-1 signaling. Mol. Psychiatry 23, 1555-1565 (2018).

14. Hsu, T. M. et al. Hippocampus ghrelin signaling mediates appetite through lateral hypothalamic orexin pathways. Elife https://doi.org/10.7554/eLife.11190 (2015).

15. Kanoski, S. E., Fortin, S. M., Ricks, K. M. \& Grill, H. J. Ghrelin signaling in the ventral hippocampus stimulates learned and motivational aspects of feeding via PI3K-Akt signaling. Biol. Psychiatry 73, 915-923 (2013).

16. Davidson, T. L. et al. Contributions of the hippocampus and medial prefrontal cortex to energy and body weight regulation. Hippocampus 19, 235-252 (2009).

17. Kanoski, S. E. \& Grill, H. J. Hippocampus contributions to food intake control: mnemonic, neuroanatomical, and endocrine mechanisms. Biol. Psychiatry 81, 748-756 (2017).

18. Davidson, T. L., Sample, C. H. \& Swithers, S. E. An application of Pavlovian principles to the problems of obesity and cognitive decline. Neurobiol. Learn. Mem. 108, 172-184 (2014).

19. Baym, C. L. et al. Dietary lipids are differentially associated with hippocampaldependent relational memory in prepubescent children. Am. J. Clin. Nutr. 99, 1026-1032 (2014).

20. Valladolid-Acebes, I. et al. Spatial memory impairment and changes in hippocampal morphology are triggered by high-fat diets in adolescent mice. Is there a role of leptin? Neurobiol. Learn Mem. 106, 18-25 (2013).

21. Boitard, C. et al. Impairment of hippocampal-dependent memory induced by juvenile high-fat diet intake is associated with enhanced hippocampal inflammation in rats. Brain Behav. Immun. 40, 9-17 (2014).

22. Boitard, $\mathrm{C}$. et al. Juvenile, but not adult exposure to high-fat diet impairs relational memory and hippocampal neurogenesis in mice. Hippocampus 22, 2095-2100 (2012)

23. Hsu, T. M. et al. Effects of sucrose and high fructose corn syrup consumption on spatial memory function and hippocampal neuroinflammation in adolescent rats. Hippocampus 25, 227-239 (2015).

24. Kendig, M. D., Boakes, R. A., Rooney, K. B. \& Corbit, L. H. Chronic restricted access to $10 \%$ sucrose solution in adolescent and young adult rats impairs spatial memory and alters sensitivity to outcome devaluation. Physiol. Behav. 120, 164-172 (2013).

25. Reichelt, A. C., Killcross, S., Hambly, L. D., Morris, M. J. \& Westbrook, R. F. Impact of adolescent sucrose access on cognitive control, recognition memory, and parvalbumin immunoreactivity. Learn Mem. 22, 215-224 (2015).

26. Noble, E. E., Hsu, T. M., Liang, J. \& Kanoski, S. E. Early-life sugar consumption has long-term negative effects on memory function in male rats. Nutr. Neurosci. 22, 273-283 (2019).

27. Walker, R. W., Dumke, K. A. \& Goran, M. I. Fructose content in popular beverages made with and without high-fructose corn syrup. Nutrition $\mathbf{3 0}$, 928-935 (2014).

28. Olson, C. A. et al. The gut microbiota mediates the anti-seizure effects of the ketogenic diet. Cell 173, 1728-1741 e1713 (2018). 
29. Martinez, M. C., Villar, M. E., Ballarini, F. \& Viola, H. Retroactive interference of object-in-context long-term memory: role of dorsal hippocampus and medial prefrontal cortex. Hippocampus 24, 1482-1492 (2014).

30. Balderas, I. et al. The consolidation of object and context recognition memory involve different regions of the temporal lobe. Learn Mem. 15, 618-624 (2008).

31. Beilharz, J. E., Maniam, J. \& Morris, M. J. Short exposure to a diet rich in both fat and sugar or sugar alone impairs place, but not object recognition memory in rats. Brain Behav. Immun. 37, 134-141 (2014).

32. Thompson, L. R. et al. A communal catalogue reveals Earth's multiscale microbial diversity. Nature 551, 457-463 (2017).

33. Caporaso, J. G. et al. Ultra-high-throughput microbial community analysis on the Illumina HiSeq and MiSeq platforms. ISME J. 6, 1621-1624 (2012).

34. Patro, R., Duggal, G., Love, M. I., Irizarry, R. A. \& Kingsford, C. Salmon provides fast and bias-aware quantification of transcript expression. Nat. Methods 14, 417-419, https://doi.org/10.1038/nmeth.4197 (2017).

35. Soneson, C., Love, M. I. \& Robinson, M. D. Differential analyses for RNA-seq: transcript-level estimates improve gene-level inferences. F1000Res 4, 1521 (2015).

36. Anders, S. \& Huber, W. Differential expression analysis for sequence count data. Genome Biol. 11, R106 (2010).

37. Kuleshov, M. V. et al. Enrichr: a comprehensive gene set enrichment analysis web server 2016 update. Nucleic Acids Res. 44, W90-97 (2016).

38. Kanehisa, M., Furumichi, M., Tanabe, M., Sato, Y. \& Morishima, K. KEGG: new perspectives on genomes, pathways, diseases and drugs. Nucleic Acids Res. 45 D353-D361 (2017).

39. The Gene Ontology, C. Expansion of the Gene Ontology knowledgebase and resources. Nucleic Acids Res. 45, D331-D338 (2017).

40. Slenter, D. N. et al. WikiPathways: a multifaceted pathway database bridging metabolomics to other omics research. Nucleic Acids Res. 46, D661-D667 (2018).

41. Aggleton, J. P. \& Brown, M. W. Contrasting hippocampal and perirhinal cortex function using immediate early gene imaging. Q. J. Exp. Psychol. B 58, 218-233 (2005).

42. Albasser, M. M., Davies, M., Futter, J. E. \& Aggleton, J. P. Magnitude of the object recognition deficit associated with perirhinal cortex damage in rats: Effects of varying the lesion extent and the duration of the sample period. Behav. Neurosci. 123, 115-124 (2009).

43. Cohen, S. J. \& Stackman, R. W. Jr. Assessing rodent hippocampal involvement in the novel object recognition task. A review. Behav. Brain Res. 285, 105-117 (2015).

44. Sestakova, N., Puzserova, A., Kluknavsky, M. \& Bernatova, I. Determination of motor activity and anxiety-related behaviour in rodents: methodological aspects and role of nitric oxide. Interdiscip. Toxicol. 6, 126-135 (2013).

45. Goran, M. I. et al. The obesogenic effect of high fructose exposure during early development. Nat. Rev. Endocrinol. 9, 494-500 (2013).

46. Noble, E. E. et al. Early-life sugar consumption affects the rat microbiome independently of obesity. J. Nutr. 147, 20-28 (2017).

47. Wang, $K$. et al. Parabacteroides distasonis alleviates obesity and metabolic dysfunctions via production of succinate and secondary bile acids. Cell Rep. 26, 222-235 e225 (2019).

48. Ling, C. et al. FAAP100 is essential for activation of the Fanconi anemiaassociated DNA damage response pathway. EMBO J. 26, 2104-2114 (2007).

49. Kim, H. S. et al. Endonuclease EEPD1 is a gatekeeper for repair of stressed replication forks. J. Biol. Chem. 292, 2795-2804 (2017).

50. Zucker, S. N. et al. Nrf2 amplifies oxidative stress via induction of Klf9. Mol. Cell 53, 916-928 (2014).

51. Yasuda, S. et al. Diacylglycerol kinase eta augments C-Raf activity and B-Raf/CRaf heterodimerization. J. Biol. Chem. 284, 29559-29570 (2009).

52. Murdoch, $\mathrm{H}$. et al. Periplakin interferes with $\mathrm{G}$ protein activation by the melanin-concentrating hormone receptor-1 by binding to the proximal segment of the receptor C-terminal tail. J. Biol. Chem. 280, 8208-8220 (2005).

53. Gerke, P. et al. Neuronal expression and interaction with the synaptic protein CASK suggest a role for Neph1 and Neph2 in synaptogenesis. J. Comp. Neurol. 498, 466-475 (2006)

54. Chen, H., Duncan, I. C., Bozorgchami, H. \& Lo, S. H. Tensin1 and a previously undocumented family member, tensin2, positively regulate cell migration. Proc. Natl Acad. Sci. USA 99, 733-738 (2002).
55. O'Mahony, A. et al. NF-kappaB/Rel regulates inhibitory and excitatory neuronal function and synaptic plasticity. Mol. Cell Biol. 26, 7283-7298 (2006).

56. Gerlai, R., Adams, B., Fitch, T., Chaney, S. \& Baez, M. Performance deficits of mGluR8 knockout mice in learning tasks: the effects of null mutation and the background genotype. Neuropharmacology 43, 235-249 (2002).

57. Shao, X. et al. HMG-CoA synthase 2 drives brain metabolic reprogramming in cocaine exposure. Neuropharmacology 148, 377-393 (2019).

58. Yang, S., Wu, P., Xiao, J. \& Jiang, L. Overexpression of COX6B1 protects against //Rinduced neuronal injury in rat hippocampal neurons. Mol. Med Rep. 19, 4852-4862 (2019).

59. Ochiai, Y. et al. The blood-brain barrier fatty acid transport protein 1 (FATP1/ SLC27A1) supplies docosahexaenoic acid to the brain, and insulin facilitates transport. J. Neurochem. 141, 400-412 (2017).

60. Kautzmann, M. I. et al. Membrane-type frizzled-related protein regulates lipidome and transcription for photoreceptor function. FASEB J. 34, 912-929 (2020).

61. David, L. A. et al. Diet rapidly and reproducibly alters the human gut microbiome. Nature 505, 559-563 (2014).

62. de La Serre, C. B. et al. Propensity to high-fat diet-induced obesity in rats is associated with changes in the gut microbiota and gut inflammation. Am. J. Physiol. Gastrointest. Liver Physiol. 299, G440-448 (2010).

63. Bruce-Keller, A. J. et al. Obese-type gut microbiota induce neurobehavioral changes in the absence of obesity. Biol. Psychiatry 77, 607-615 (2015).

64. Leigh, S. J., Kaakoush, N. O., Westbrook, R. F. \& Morris, M. J. Minocyclineinduced microbiome alterations predict cafeteria diet-induced spatial recognition memory impairments in rats. Transl. Psychiatry 10, 92 (2020).

65. Leigh, S. J., Kaakoush, N. O., Bertoldo, M. J., Westbrook, R. F. \& Morris, M. J. Intermittent cafeteria diet identifies fecal microbiome changes as a predictor of spatial recognition memory impairment in female rats. Transl. Psychiatry 10, 36 (2020).

66. Li, J. M. et al. Dietary fructose-induced gut dysbiosis promotes mouse hippocampal neuroinflammation: a benefit of short-chain fatty acids. Microbiome 7, 98 (2019).

67. Alamy, M., Errami, M., Taghzouti, K., Saddiki-Traki, F. \& Bengelloun, W. A. Effects of postweaning undernutrition on exploratory behavior, memory and sensory reactivity in rats: implication of the dopaminergic system. Physiol. Behav. 86, 195-202 (2005).

68. Kendig, M. D., Westbrook, R. F. \& Morris, M. J. Pattern of access to cafeteria-style diet determines fat mass and degree of spatial memory impairments in rats. Sci. Rep. 9, 13516 (2019)

69. Yang, Y. et al. Early-life high-fat diet-induced obesity programs hippocampal development and cognitive functions via regulation of gut commensal Akkermansia muciniphila. Neuropsychopharmacology 44, 2054-2064 (2019).

70. Takeuchi, T. et al. Locus coeruleus and dopaminergic consolidation of everyday memory. Nature 537, 357-362 (2016).

71. Kempadoo, K. A., Mosharov, E. V., Choi, S. J., Sulzer, D. \& Kandel, E. R. Dopamine release from the locus coeruleus to the dorsal hippocampus promotes spatial learning and memory. Proc. Natl Acad. Sci. USA 113, 14835-14840 (2016).

72. Azevedo, E. P. et al. A role of Drd2 hippocampal neurons in contextdependent food intake. Neuron 102, 873-886 e875 (2019).

73. Ou, Z. et al. Protective effects of Akkermansia muciniphila on cognitive deficits and amyloid pathology in a mouse model of Alzheimer's disease. Nutr. Diabetes 10, 12 (2020).

74. Hu, L. et al. High salt elicits brain inflammation and cognitive dysfunction, accompanied by alternations in the gut microbiota and decreased SCFA production. J. Alzheimers Dis. 77, 629-640 (2020).

75. Stanhope, K. L. Sugar consumption, metabolic disease and obesity: the state of the controversy. Crit. Rev. Clin. Lab. Sci. 53, 52-67 (2016).

76. Cerdo, T., Dieguez, E. \& Campoy, C. Early nutrition and gut microbiome: interrelationship between bacterial metabolism, immune system, brain structure, and neurodevelopment. Am. J. Physiol. Endocrinol. Metab. 317, E617-E630 (2019).

77. MahmoudianDehkordi, S. et al. Altered bile acid profile associates with cognitive impairment in Alzheimer's disease-an emerging role for gut microbiome. Alzheimers Dement. 15, 76-92 (2019). 